\title{
Research on university faculty members' reasoning about how departments change
}

\author{
Gina M. Quan, ${ }^{1}$ Joel C. Corbo, ${ }^{1}$ Courtney Ngai, ${ }^{2}$ Daniel Reinholz, ${ }^{3}$ and Mary E. Pilgrim ${ }^{3}$ \\ ${ }^{1}$ Center for STEM Learning, University of Colorado Boulder, Boulder, CO, 80309 \\ ${ }^{2}$ The Institute of Learning and Teaching, Colorado State University, Fort Collins, CO, 80523 \\ ${ }^{3}$ Department of Mathematics and Statistics, San Diego State University, San Diego, CA, 92182
}

\begin{abstract}
Research on institutional change says that effective change agents are able to flexibly reason with multiple perspectives on change, depending on their local context and their goals. However, little is known about what this flexible reasoning looks like. In this exploratory work, we conducted and analyzed interviews in which faculty discussed departmental change. This work is part of an ongoing study to understand how to support departmental change through Departmental Action Teams (DATs). Our preliminary analyses suggest that faculty have multiple context-dependent ways to reason about change. This work will lead to a better understanding of how productive lines of reasoning can be leveraged in faculty communities that are trying to create change.
\end{abstract}

\section{INTRODUCTION}

Understanding how to create lasting, sustainable change to educational practices has been a central goal in physics education research $[1,2]$. Often, the cultural features of university departments (e.g., their norms, values, rewards structures) can inhibit or support the adoption of change efforts, particularly efforts aimed at individual courses or individual faculty [3]. This motivates a focus on understanding change at the scale of the department, rather than the scale of individuals.

Our research team supports change through Departmental Action Teams (DATs), departmentally-based working groups that aim to sustainably improve undergraduate STEM education [2, 4]. Our programmatic goals are twofold: 1) support DATs in creating sustainable changes to their departments and 2) build capacity within DAT members to become agents of future change. To enact our second goal, it is necessary for us to understand how DAT members think about change.

In her book How Colleges Change, Kezar outlines six perspectives that successful change agents in higher education use [3]. Each perspective illustrates different mechanisms by which change happens. These perspectives can be used to characterize change and to inform change agents' approaches toward change efforts. Kezar claims that skillful change agents are able to consider multiple change perspectives and determine which one(s) are appropriate to the context and type of change desired.

However, there has yet to be research illustrating how individuals reason with those change perspectives. Such an understanding is valuable to those who study change in higher education and those who want to facilitate the development of change agents. Within this paper, we provide exploratory analyses of how departmental change agents-specifically faculty-reason about how change happens. Our research questions are:

1. How do faculty reason in ways that are aligned with the change perspectives outlined by Kezar?

2. How are these lines of reasoning dependent on context?
TABLE I. List of key components of each change perspective

\begin{tabular}{l|l}
\hline \hline Perspective & The mechanisms by which change emerges \\
\hline Scientific Management & Incentives and rewards \\
Social Cognition & Shifts in individuals' understanding \\
Cultural & Shifts in/alignment with culture \\
Evolutionary & External, unplanned factors \\
Political & Collective action and bargaining \\
Institutional & Pressures from other institutions \\
\hline \hline
\end{tabular}

\section{THEORETICAL PERSPECTIVE}

We list Kezar's six perspectives in Table 1. Within a single change perspective, there is a multifaceted set of approaches toward change and assumptions about how change happens. Effective change agents both 1) reason with a multiplicity of perspectives and 2) determine which perspective(s) are appropriate to the context and type of change desired. We use Kezar's framework because her work is tailored to highereducation.

This paper focuses on the three perspectives that were most common in our data. Scientific management assumes that change is driven by the leaders of an organization, who shift incentives, rewards, and structures to make change happen. Social cognition assumes that change comes from individuals' shifts in understanding and that resistance to change comes from lack of understanding. To create change, one should support others' learning. Cultural focuses on whether a change effort aligns with existing aspects of culture (values, beliefs, norms); the more aligned the effort is with the existing culture, the more likely it is to succeed.

To characterize faculty thinking, we adopt a situated resources perspective, which assumes individuals hold many productive ideas to reason about any given topic [5, 6]. Such conceptual ideas dynamically interact with context, identities, epistemologies, and emotions [7,8]. While a situated resources perspective has been developed to study student's conceptual reasoning about physics, we find it useful to apply to faculty because it foregrounds the multiplicity of ideas 
they hold. We use this perspective to specifically understand how faculty discuss change, which draws our attention to how context and emotion mediate their reasoning.

\section{ANALYTICAL APPROACH}

This study is embedded within a larger research project on Departmental Action Teams (DATs) [2, 9]. DATs are composed of faculty, students, and/or staff within a single department, working toward enacting sustainable changes. External facilitators support DAT members in enacting successful changes and developing capacity as change agents. The DAT model was created and refined in two large universities.

This paper considers a DAT in a natural sciences department that we refer to as Potions. The Potions DAT focused on the recruitment and retention of women and underrepresented minorities (for more details, [9, 10]). The initial DAT comprised of four faculty members, one postdoc, and two postdocs as external facilitators (both facilitators are authors). After year one, an external researcher (not a facilitator) collected semi-structured one-on-one interviews with all DAT members who weren't facilitators (five interviews total). Interviews focused on participants' views of DAT outcomes, the role of facilitation, and their views about departmental change. Audio recordings of interviews were transcribed.

Our analysis began by reading transcripts and identifying segments where DAT members discussed how change happened. We narrowed our analyses to the four faculty interviews because faculty on the Potions DAT had the most detailed descriptions of the departmental history and context. We chunked the transcripts [11] by unique "stories" in which the interviewee described a single change effort. The first two authors independently open-coded those chunks for alignment with one of Kezar's six perspectives, and reached consensus on codes. Finally, we created analytic memos [11] and progressively refined our analyses [12]. Across the four interviews, the change perspectives that faculty most commonly reasoned with were cultural, social cognition, and scientific management. We drew upon the facilitators' and researchers' experience in the Potions DAT and other natural sciences departments to inform our interpretations [13], particularly interpretations related to the cultural perspective.

In the next section, we show data from two interviewees, Sally and Alex, who are tenure track faculty in Potions. Both Sally and Alex used two similar stories to characterize change efforts: 1) that scientific management is ineffective because of cultural logic, and 2) that social cognition is effective because of cultural logic. We selected these data because they were common across our data set (story 1 occurred in four interviews and story 2 occurred in three interviews) and because their transcripts were richly detailed. Our purpose in this paper is to provide examples of how faculty reason about change; therefore, we do not endorse or critique the productivity of these lines of reasoning within this paper. Moreover, while these lines of reasoning were common across the data set, we do not make claims about their representativeness across populations of faculty.

\section{RESULTS}

Now we present the stories that Sally and Alex used to describe change efforts within their department, including those outside of the DAT. We highlight commonalities and differences in their reasoning patterns and accounts.

\section{A. Story 1: Scientific Management failed because of misalignment with culture}

The first story is that top-down approaches (i.e., scientific management) are ineffective because of Potions culture. Sally describes the individualistic nature of Potions, which leads faculty to value individuals' freedom. This cultural feature leads faculty to resist scientific management approaches.

Sally: When I first came there was... some number of faculty who were very resistant to the idea of being told to change the way that they teach. I remember people making comments about I'm not going to use clickers or, you know, do whatever, just because [leader in STEM education] says that's what I should do, you know? This principle of academic freedom and choosing how you're going to do your research and teach your class can become sort of a tool that people use to resist doing anything differently.

In this segment, Sally characterizes prior teaching reform efforts as consisting of the faculty "being told to change" their teaching practices, often by an authority figure. These topdown descriptions are aligned with a scientific management perspective of change. She claims that such approaches elicit resistance due to a "principle of academic freedom," which she describes as "choosing how you're going to do your research and teach your class." In and of itself, this cultural feature is not incompatible with making changes to teaching. However, in the context of a top-down change, Sally sees her colleagues as choosing to use it as a tool to resist change.

Alex similarly argues that individualism is a source of resistance to scientific management changes.

Alex: There was a history in the [change effort to improve instruction]... to say that the [change effort] would develop courses that we would then be required to teach. And faculty-I mean, we're not allowed to park on campus anymore as faculty members unless we pay the going rate. The last thing we can do is teach the damn class the way we want to teach it. So when somebody says, yeah, we're going to make a standard that you'll have to adhere to...It's just a terrible idea. And yet just exactly that language was used early on 
in the [change effort]. Including teeth during performance evaluation, that if your class didn't adhere to the defined class that you would receive a substandard rating and raise.

Alex describes several features of the scientific management perspective, including the requirement to teach in certain externally-defined ways and the use of punitive measures ("a substandard rating and raise") to incentivize change. He characterizes this approach as "terrible," citing similar ideas to what Sally called "academic freedom" and comparing it to unpleasant administrative policy changes. In the excerpt from Alex, he also has a strong emotional reaction toward the idea of being told how to teach; this is different from Sally, who depersonalized the issue by framing the use of academic freedom as something that "people" do to resist change.

Within these two excerpts, we see that scientific management was viewed negatively by both interviewees as a way to make change in their department. Sally described how faculty invoke the cultural feature of "academic freedom" to resist change; Alex also recognizes academic freedom as a reason to resist change but additionally connects top-down changes that impact teaching to a history of feeling increasingly restricted by other policies (e.g., parking fees). In both cases, Sally and Alex use a cultural perspective to explain why some scientific management approaches do not work in their department. According to them, a cultural feature of Potions is a sense of individualism: they value independence and freedom of action. Similar rhetoric has been used in academia more broadly (c.f., [14]). This individualism manifests in Sally's and Alex's stories and it is misaligned with a scientific management change effort that tries to function through adherence to standards, appeals to authority, and punishments. Thus, Sally and Alex utilize a cultural perspective to explain how the cultural features of Potions cause a scientific management change effort to be ineffective.

\section{B. Story 2: Social Cognition works because of alignment with culture}

In the next change story, participants discuss the alignment of cultural features of Potions with a social cognition approach to change. Immediately after the above excerpt, Sally presents a successful approach to improving teaching:

Sally: I think one thing that's made [change] happen is people seeing how it works and seeing examples of other people doing it and sort of imitating their colleagues, and also becoming more familiar with the research that this can be beneficial... For [Potions] faculty, seeing examples of success and seeing data, my impression is that those are the things that tend to be persuasive.

In this excerpt, Sally describes activities that supported faculty in changing their teaching practices, including "seeing how it works," testing it out themselves, "seeing data," and "becoming more familiar with the research." Sally draws on the social cognition perspective, which claims that resistance to change comes from lack of understanding the change [3]. According to this perspective, using evidence to shift thinking is a key mechanism to create change. In the last sentence of the excerpt, Sally explicitly states that these forms of evidence she listed are "persuasive" to Potions faculty. We interpret this sentence to mean that she thinks data is particularly compelling for Potions faculty because it aligns with the Potions cultural feature of valuing data. Scientists often say that they value using data to generate understanding (c.f., [15]).

Alex also frames a social cognition perspective as aligning with Potions cultural features, although he describes different cultural features than Sally. Specifically, Alex draws upon the idea that Potions faculty are knowledge-seekers:

Alex: Just like any other field, [Potions] sees itself as progressive, so we're trying to break new ground regularly...I think that the [Potions] department has a long history of being concerned about what we're doing right and what we're doing wrong. We don't have as long a history about being quantitative about it...And it is confusing to wonder why the vast majority of half of humanity doesn't see [Potions] as an interesting area to go into. That we hover in the low teens in terms of number of our students who are female... There's something going on there, everybody knows that there's got to be something going on there. But we don't honestly think we know what it is... I think that's something that thinking people want to understand...

Alex starts out by describing cultural features of Potions: that it is "progressive" and "trying to break new ground." He uses these features of Potions culture explain why Potions cares about representation and recruitment, which was the focus of their DAT. Alex argues that the Potions DAT's mission of improving representation and recruitment of underrepresented students is aligned with Potions culture; thus, he is using the cultural perspective to reason why he would expect the DAT to be successful. Alex then reasons in ways aligned with the social cognition perspective. His description of "thinking people" wondering about confusions and wanting to know what's "going on there" is aligned with the social cognition practice of using cognitive conflict to drive deeper understanding. In this case, Alex uses "we" to describe the cognitive conflict and the subsequent drive to understand. The use of "we" suggests that Alex sees knowledge-seeking behavior as characteristic of a Potions culture.

Alex then describes why he values the DAT approach:

Alex: We've got a committee that's going to make suggestions and keep track of numbers [of retention data], and if the numbers become overwhelming, and we think there's a path to understanding, then things will change. 
Alex describes the DAT as focusing on tracking "numbers" and identifying a "path to understanding," which he says will lead to change. This focus on deeper understanding is consistent with the social cognition perspective and the cultural feature of Potions-faculty-as-knowledge-seekers from above.

Sally and Alex both reason with the cultural perspective by identifying aspects of Potions culture that support a social cognition approach to change. Sally highlights how Potions faculty are persuaded by data and evidence, because Potions values data. This line of reasoning came up in all four Potions faculty interviews. Alex also described additional $\mathrm{cul}$ tural features of Potions - that Potions faculty value seeking knowledge - to explain why Potions faculty seek answers to troubling questions such as representation and recruitment. Resolving cognitive conflict and using data are classic components of a social cognition perspective.

\section{DISCUSSION}

We have illustrated two stories of change efforts that emerged in faculty interviews: scientific management change as ineffective because of misalignment with cultural values of individualism, and social cognition change as effective because of alignment with cultural values of data analysis and understanding. This exploratory work demonstrates how some faculty reason about change in university departments.

This will inform future analyses of how different reasoning patterns emerge and impact faculty members' in-the-moment decision-making. While our purpose in this paper is not to endorse or critique these lines of reasoning, we plan to research which lines of reasoning are most productive for change agents to use. This will deepen our field's understanding of how to nurture effective change. Future work will discuss additional ways faculty use change perspectives to reason about change.

This work is helpful for the facilitation of change efforts. Understanding the common ways faculty reason about change, as well as how those lines of reasoning may be lever- aged, can support facilitators in guiding discussion. For example, if a facilitator notices a particular change perspective is relevant to a conversation, they can encourage participants to reason with that change perspective. Categorizing which change perspectives faculty are using is useful toward identifying which change perspectives are not being used, and facilitators can encourage faculty to utilize alternative perspectives. We now teach DAT members about different change perspectives to encourage their awareness and evaluation of their own thinking about change. Such judgements are necessary toward becoming effective change agents [3].

We put forth these characterizations of faculty thinking to highlight the rich ways that faculty think about change. In our informal and formal discourse, our field often uses deficit and unitary labels to describe faculty, with some exceptions (e.g., [16, 17]). For example, some university instructors become labeled as "set in their ways" while others are thought of as "low hanging fruit." Such language implies that instructors either hold "correct" or "incorrect" notions about change. Our research shows that faculty have many different ideas about change, which are more or less productive given the context. This parallels discussions of student thinking, where we have found that students have many seeds of productive ideas and our instruction should leverage those seeds (rather than assuming that students have "incorrect" ideas about physics that instructors must replace) [6]. Thus, we argue that there is value in considering faculty thinking about change in the ways that parallel consideration of student thinking about physics. Our work adds nuance the field's understanding of faculty thinking by describing the ideas they bring to understanding how change happens in departments.

\section{ACKNOWLEDGMENTS}

We thank the DAT Project Team, Dimitri Dounas-Frazer, Andrew Elby, and Laura Ríos for their thoughtful contributions. This material is based upon work supported by the National Science Foundation under Grant No. 1626565.
[1] C. Henderson, A. Beach, and N. Finkelstein, J. Research Sci. Teach. 48, 952 (2011), ISSN 1098-2736.

[2] J. C. Corbo, D. L. Reinholz, M. H. Dancy, S. Deetz, and N. Finkelstein, Phys. Rev. Phys. Educ. Res. 12, 010113 (2016).

[3] A. Kezar, How colleges change: Understanding, leading, and enacting change (Routledge, 2013).

[4] Reinholz, D. L., Corbo, J. C., Dancy, M., and Finkelstein, N., Learn. Communities J. 9 (2017).

[5] A. Gupta and A. Elby, Int. J. of Sci. Ed. 33, 2463 (2011).

[6] D. Hammer, Am. J. Physics 68, S52 (2000), ISSN 0002-9505.

[7] L. Lising and A. Elby, Am. J. Physics 73, 372 (2005).

[8] A. Gupta, A. Elby, and B. A. Danielak, Phys. Rev. Phys. Educ. Res. 14, 010129 (2018).
[9] J. Corbo, D. L. Reinholz, and M. H. Dancy, 2015 PERC Proceedings (2015).

[10] K. Rainey, J. Corbo, and B. M. Reinholz, D, 2016 PERC Proceedings pp. 264-267 (2016).

[11] B. Jordan and A. Henderson, J. Learn. Sci. 4, 39 (1995).

[12] R. A. Engle, F. R. Conant, and J. G. Greeno, Video research in the learning sciences pp. 239-254 (2007).

[13] H. S. Becker and B. Geer, Human Organization 16, 28 (1957).

[14] J. Schmidt, Disciplined minds (Rowman \& Littlefield, 2001).

[15] N. G. Lederman, J. Res. Sci. Teach. 29, 331 (1992).

[16] D. M. Levin, D. Hammer, and J. E. Coffey, J. Teach. Ed. 60, 142 (2009).

[17] A. Olmstead and C. Turpen, Phys. Rev. Phys. Educ. Res. 13, 020123 (2017) 